\title{
Using Health Belief Model to Probe Female Adolescent Perception About Breast Cancer in Riyadh City
}

\author{
Manal Fehade Al-Harbi*, Kholoud Al-Haji, Salma Abd El Atty Moawed, Abeer Hawsawi \\ Maternity \& Child Health Nursing Department, College of Nursing, King Saud University, Riyadh, Kingdom of Saudi Arabia
}

\author{
Email address: \\ drmanalalharbi@gmail.com (M. F. Al-Harbi) \\ ${ }^{*}$ Corresponding author
}

\section{To cite this article:}

Manal Fehade Al-Harbi, Kholoud Al-Haji, Salma Abd El Atty Moawed, Abeer Hawsawi. Using Health Belief Model to Probe Female Adolescent Perception About Breast Cancer in Riyadh City. American Journal of Nursing Science. Vol. 6, No. 4, 2017 , pp. 358-365. doi: 10.11648/j.ajns.20170604.20

Received: June 19, 2017; Accepted: June 28, 2017; Published: July 24, 2017

\begin{abstract}
The perceived of those susceptible to breast cancer (BC) such as female adolescent is significant. Early detection lead to early prevention. The present study aimed to probe the perception of female adolescent at age group of 15-20 years in Riyadh about BC (phenomena) and the relationship between demographic characteristics of students and their perception regarding $\mathrm{BC}$ as a way of improving high school student toward health knowledge. A cross-sectional prospective study conducted among governmental and private school students. The study was conducted in Riyadh, Saudi Arabia from February to May 2014. A Representative probability sample of secondary school girls $(n=917)$ in both Governmental and private was recruited for conducting this study. A structured questionnaire was developed which included part about four constructs of Health Belief Model and other parts about sociodemographic data and items related to students' source of information about $\mathrm{BC}$. The validity and reliability for the scale was established. It was found that the perceived level of participants was moderate $(\mathrm{M}=3 ; \mathrm{SD}=1.07)$ which provide an important baseline data about perception of toward BC. It is crucial to improve students level of perception toward BC and practice of breast self-examination (BSE) among female adolescent students within community. A further study is needed to explore the perception of female adolescent using both quantitative and qualitative methodology to elicit cultural issue in BC perception and breast self-exam.
\end{abstract}

Keywords: Breast Cancer, Breast Self-exam, Female, Adolescent, Health Belief Model

\section{Introduction}

The American medical association report on Global Burden of Disease Cancer indicated that Cancer mortality is high and considered the second leading cause of death worldwide. Cancer cases increased by $33 \%$ including $16 \%$ as population aging, $13 \%$ as population growth and $4 \%$ as changes in age-specific rates [1]. While prostate cancer (1.6 million cases) was the most common cancer for men, Breast cancer (2.4 million cases) was the most common cancer for women world widely. Breast cancer (BC) is among most invasive caners and the most common cancer in American women, except for skin cancers and the second leading cause of cancer death in women [2]. In the United States (US) the center for disease control and disease prevention center (CDC) reported a total of $1,559,130$ invasive cancers were diagnosed [3]. According to the American Cancer Society's (ACS) an estimated of 252,710 new cases of invasive BC will be diagnosed in women and 40,610 women will die from $\mathrm{BC}$ also the trend of $\mathrm{BC}$ is common equally between white and African and less common among other races/ethnicities [2]. As most of $\mathrm{BC}$ deaths occur in developing countries, two thirds of $\mathrm{BC}$ deaths occur in low-income and middle-income countries (LMICs) where $\mathrm{BC}$ is diagnosed in very late stage due to lack of diagnostic and treatment services such as mammography and radiotherapy [4]. Survival of women who develop BC in LMICs is low; the opposite is true for women in a high-income country [5]. The incidence rate of $\mathrm{BC}$ is increasing in rich developing country due to increase life expectancy, increase urbanization and adoption of western lifestyles [6]. In Saudi Arabia (SA) which is a rich Arab gulf country $\mathrm{BC}$ is high prevalence type of cancer which accounting for about $11 \%$ of all women cancer cases [7]. 
The more recent report by Saudi general authority of statistics showed $43 \%$ of total population was female [8] which indicate the required need to investigate their perception toward $\mathrm{BC}$. Therefore, the current study utilized health belief model (HBM) like other studies used HBM to predict behavior toward breast cancer [9-10]. Healthbehavior theories assist researchers by organizing their inquiry into why people do or do not engage in specific health behaviors and explain behavior and suggest ways to achieve behavior change [11-12]. HBM has 6 concepts (Susceptibility: perceived personal vulnerability to or subjective risk of a health condition, Seriousness: perceived degree of personal harm from the condition, Benefits: perceived positive attributes of an action, and Barriers: perceived negative aspects related to an action) to stipulate that health-related behavior as influenced by a person's perception of the threat posed by a health problem and by the value associated with his or her action to reduce that threat[13-14]. A cross-sectional, community-based study utilized HBM to measure family history of BC, worry about getting $\mathrm{BC}$, overall knowledge about $\mathrm{BC}$ risk factors, as well as women's perceived susceptibility of getting $\mathrm{BC}$ the findings reveled family history and $\mathrm{BC}$ worry were found to be significant predictors of personal perception for risk judgments [15]. Another interventional Turkish study used Health Belief Model Scale (CHBMS) which comprised a total of 42 items with six subscales, revealed that the perceived benefits and confidence related to BSE increased and perceived barriers decreased significantly after intervention [16]. Therefore, the current paper aims to examine the perception of female adolescent about BC. Thus, the present study more specifically aimed to 1) Measure the perception level of female secondary school students about $\mathrm{BC}$ using HBM and 2) Identify the relationship between demographic factors and perception of female secondary school students about BC.

\section{Methods}

\subsection{Design \& Setting}

A descriptive cross-sectional design was utilized to study the perception of female adolescent about breast cancer (phenomena) and the relationship between variables (demographic characteristics of students and their perception regarding breast cancer). The study was conducted over 4 months at governmental and private secondary girls' schools and completed May 2014. A representative probability sample of secondary school girls was recruited for conducting this study. The selection of the schools stratified from the total population within five educational regions in Riyadh city.

\subsection{Sample \& Sampling Technique}

The sample size was calculated to measure an expected satisfactory perception rate of $50 \%$ or higher, with absolute precision $5 \%$ ( $10 \%$ of the prevalence), and a design effect 2 due to multistage sampling. Using a sample size equation for a single proportion (EpiInfo 6.04) at a 95\% level of confidence, the required sample size would be 769 girls. This sample was increased to 900 to compensate for a dropout rate of about $15 \%$. The inclusion criteria were secondary school girls and excluded those participants who were absent or couldn't complete the questionnaire for any reason. A multistage stratified sampling technique was used. The first stage involved selection of the schools stratified from the total population (students) in the five educational regions. Those five regions have two types of schools governmental $(n=142)$ and private $(\mathrm{n}=109)$. Then a proportionate sample (two regions) was selected randomly which include (governmental schools $=7 \&$ private schools $=5$ ). The second stage involved selection of classes from selected schools $(n=12)$. From each selected school, 3 classes were selected randomly, one from each grade $(n=36)$ governmental classes were 21 classes and 15 private class. Therefore, the third stage consisted of recruitment of students from the selected classes. All students in the selected classes were recruited. With an assumed average of 25 students per class, the required sample size was achieved from the selected 36 classes.

\subsection{Data Collection Tools}

A self-administered questionnaire was developed after the review of literature with two parts. Part one sociodemographic characteristics such as age, secondary school type, marital status, personal and family history for BC. The questions composed answers of "Yes, No, or I don't know". Part two perception of students about BC and BSE. It was assessed by a scale that is composed of 26 questions [17] which was measured through use the health belief model (HBM), the statements of the HBM variables is (perceived susceptibility, perceived severity, perceived benefits and perceived barriers). The current scale was developed based on the work of many researchers [18-19].

On the other hand, the modified HBM for BC instrument included 26 items on four constructions, perceived susceptibility - "SUS" (3 items), perceived severity - "SER" (7 items), perceived benefits - "BEN" (5 items), perceived barriers "BAR" (11 items). Participants rated these statements using a five-point Likert scale (strongly agree, agree, undecided, disagree, strongly disagree) each statement was scored from 1 to 5 where 5 indicates strong or higher perception and 1 indicates weak or lower perceptions.

\subsection{Reliability and Validity}

The Reliability and internal consistency was measured for the present study. The result for overall scale was $(0.7)$. To establish content validity, the questionnaire was sent to panel of six experts (two nursing faculty members, two oncology doctors from the oncology department of King Saud Medical Complex, one doctor from the Maternity Hospital, and one from the family health department reviewed the questionnaire for content validation and minor changes were recommended. 


\subsection{Pilot Study}

A convenience sample of 20 high school female students (excluded from the final study) was considered for pilot testing to test the internal consistency of the items and check the feasibility of the instrument. Internal consistency reliability was tested using Cronbach alpha on appropriate sections of all returned surveys. The pilot study helped to accommodate the aim of the work to actual feasibility, identify the difficulties may be faced during the application, and to study all the procedures and activities of the administrative aspects. Also, the time of completing the questionnaire was estimated during this pilot study to be 15 $\min$. The necessary modifications according to the results obtained were done, so some statements were re-constructed. Also, the structure of the questionnaire sheet was reformatted to facilitate data collection for the main study.

\subsection{Ethical Considerations}

The study has no real or potential harm for the study participants. Participants confidentially ensured and participation is completely voluntary. The researchers respected the autonomy of the participants and informed them for the right to participate and withdraw any time. Also their participation or non-participation in the study would have no impact on their degree. After getting the approval from the ethical committees, data were collected between February to May 2014. The participants were informed by the researchers about the objectives of the study, confidentiality of their responses and the benefits. Written informed consent was obtained from each respondent. An official written permission was obtained from the human ethics committee in Ministry of Education, Department of Girls' Education, Riyadh Region to carry out this study in 12 schools.

\subsection{Data Analysis}

Analysis of data was done using SPSS 16.0 statistical software. Descriptive statistics such as frequencies, range of frequencies, percentages, means, and standard deviations was used to describe demographic data of participants. In addition, to measure the scale items analysis coefficient of variation $\left(\mathrm{C}_{\mathrm{V}}\right)$ was useful for comparison between data sets with different means. The actual value of the $\left(\mathrm{C}_{\mathrm{V}}\right)$ is independent of the unit to be used instead of the standard deviation.

\section{Results}

The rate of drop-out among participants (attrition rate) was $10 \%$ only. Out of the 1000 questionnaires administered to the participants, 917 were returned the questionnaires. In this study Cronbach's alpha was computed to examine the internal consistency for overall scale was (0.7).
Table 1. Distribution of the female students' socio-demographic Characteristics $(n=917)$.

\begin{tabular}{llll}
\hline & Category & No & \% \\
\hline \multirow{3}{*}{ Age } & $15-16$ years & 328 & 36 \\
& 17-18 years & 491 & 54 \\
Secondary School Type & 19-20 years & 84 & 9.0 \\
& Private & 302 & 33 \\
Nationality & Governmental & 615 & 67 \\
& Saudi & 749 & 82 \\
Marital Status & Non-Saudi & 168 & 18 \\
& Single & 899 & 98 \\
Secondary school levels & Married & 18 & 2.0 \\
& First & 268 & 29 \\
& Second & 290 & 32 \\
Family income & Third & 359 & 39 \\
& Good & 31 & 3.0 \\
& Medium & 540 & 59 \\
& Poor & 346 & 38 \\
\hline
\end{tabular}

Table 1 shows $54 \%$ aged between $17-18$ years, $36 \%$ were aged between 16 and 17 years and $9 \%$ were aged between 19 and 20 years old. For school type $67 \%$ were governmental and $33 \%$ were private. The majority were Saudi $82 \%$, single $98 \%$, married $20 \%$ and $18 \%$ were nonSaudi. For high school levels; $1^{\text {st }}$ secondary level $29 \%, 2^{\text {nd }}$ secondary level $32 \%$ and the $3^{\text {rd }}$ secondary level $39 \%$ of the total sample. For family income $59 \%$ considered their financial status as medium, $38 \%$ as poor, and $3 \%$ as good.

Table 2. Distribution of participants according to Personal \&Family History for $B C(n=917)$.

\begin{tabular}{llll}
\hline Questions & Categories & No & \% \\
\hline \multirow{2}{*}{$\begin{array}{l}\text { Do you complain of problems in } \\
\text { the breasts? }\end{array}$} & Yes & 72 & 8.0 \\
& No & 723 & 79 \\
& I don't know & 122 & 13 \\
Have you had any breast surgery? & Yes & 23 & 3 \\
& No & 887 & 97 \\
& I don't know & 7 & 1.0 \\
Does a family member & Yes & 58 & 6.0 \\
suffer/suffered from BC? & No & 816 & 89 \\
& I don't know & 43 & 5.0 \\
Does a family member have any & Yes & 107 & 12 \\
type of cancer? & No & 727 & 79 \\
& I don't know & 83 & 9.0 \\
\hline
\end{tabular}

Table 2 presents the personal and family history of BC summarized in four questions. More than two third $79 \%$ of the sample did not complain of any breast problems whilst $8 \%$ complained from breast problems. The majority of the participants 97\% answered with a "no" while 3\% participants answered "yes" for the third question. $89 \%$ of the participants have a negative family history of $\mathrm{BC}$, and other cancer $79 \%$. While $6 \%$ had a positive family history of $\mathrm{BC}, 12 \%$ participants had a positive family history of other types of cancers. 
Table 3. Participants perception Level about Breast Cancer.

\begin{tabular}{|c|c|c|c|c|c|}
\hline Items & $\begin{array}{l}\text { Strongly } \\
\text { agree }(\%)\end{array}$ & Agree (\%) & Neutral (\%) & $\begin{array}{l}\text { Disagree } \\
(\%)\end{array}$ & $\begin{array}{l}\text { Strongly } \\
\text { Disagree (\%) }\end{array}$ \\
\hline \multicolumn{6}{|l|}{ Perceived susceptibility } \\
\hline 1.Because I am a woman susceptible to BC & 21 & 36 & 27 & 6.4 & 10 \\
\hline 2.Compare my health to other, I feel more injury to $B C$ than others & 3 & 8 & 34 & 37.3 & 19 \\
\hline 3.Increasing the possibility of $B C$, with advance the age & 11 & 15 & 40 & 24.1 & 10 \\
\hline Subscale items statistics & 12 & 19 & 33 & 23 & 13 \\
\hline \multicolumn{6}{|l|}{ Perceived severity } \\
\hline 5.BC dangerous to my health & 61 & 27 & 7 & 4 & 1 \\
\hline 6.I am permanently concerned of $B C$ & 20 & 19 & 25 & 25 & 10 \\
\hline 7.BC possible that threatens your future educational and practical & 28 & 26 & 22 & 17 & 8 \\
\hline 8.BC adversely affects the physical conditions of the patient & 19 & 20 & 35 & 13 & 12 \\
\hline 9.BC threatens the marital and family relations & 24 & 21 & 33 & 13 & 9 \\
\hline 10.BC as possible causes of death in the absence of early treatment & 47 & 27 & 20 & 3 & 3 \\
\hline Subscale items statistics & 35 & 25 & 22 & 12 & 7 \\
\hline 12.BSE helps early detection of $B C$ & 56 & 27 & 13 & 2 & 1 \\
\hline 13.Early detection leads less surgical intervention and best healing & 50 & 26 & 19 & 3 & 2 \\
\hline 14.BSE is an effective method for detecting cancer & 37 & 38 & 21 & 3 & 1 \\
\hline 15.Procedural BSE regularly it reduces the fear of $B C$ & 39 & 37 & 18 & 4 & 2 \\
\hline Subscale items statistics & 45 & 32 & 18 & 3 & 1 \\
\hline \multicolumn{6}{|l|}{ Perceived barriers } \\
\hline 16.I do not find time to perform BSE & 10 & 17 & 37 & 26 & 11 \\
\hline 17.I do not see the importance to conduct BSE & 5 & 6 & 26 & 38 & 25 \\
\hline 18.I do not know the correct way to conduct BSE & 28 & 34 & 15 & 15 & 8 \\
\hline 19.BSE increases my anxiety and fear & 23 & 19 & 22 & 26 & 11 \\
\hline 20.Not conducted BSE because of fear of cancer is detected & 12 & 14 & 27 & 31 & 17 \\
\hline 21.I do not check because my age, is an exhibition of cancer & 9 & 7 & 25 & 34 & 25 \\
\hline 22.I do not check because no one from the family has had cancer & 9 & 10 & 28 & 29 & 25 \\
\hline 23.I do not check for that defect & 4 & 3 & 17 & 34 & 42 \\
\hline 24.I do not check because it is discomfort & 4 & 5 & 18 & 41 & 32 \\
\hline Subscale items statistics & 12 & 12 & 24 & 31 & 21 \\
\hline
\end{tabular}

Table 3. Participants perception Level about Breast Cancer.

\begin{tabular}{|c|c|c|c|c|}
\hline Items & $\begin{array}{l}\text { Standard } \\
\text { Deviation }\end{array}$ & Mean & $\begin{array}{l}\text { Coefficient of } \\
\text { Variance }\left(C_{V}\right) \\
\end{array}$ & Rank \\
\hline \multicolumn{5}{|l|}{ Perceived susceptibility } \\
\hline 1.Because I am a woman susceptible to BC & 1.17 & 3.5 & 0.33 & 9 \\
\hline 2.Compare my health to other, If feel more injury to $B C$ than others & 972 & 2.3 & 0.41 & 16 \\
\hline 3.Increasing the possibility of $B C$, with advance the age & 1.10 & 2.9 & 0.38 & 13 \\
\hline Subscale items statistics & 1.08 & 2.9 & & \\
\hline \multicolumn{5}{|l|}{ Perceived severity } \\
\hline 4.I feel of fear from injury of $B C$ & 1.05 & 4.0 & 0.26 & 8 \\
\hline 5.BC dangerous to my health & .882 & 4.4 & 0.20 & 1 \\
\hline 6.I am permanently concerned of $B C$ & 1.28 & 3.1 & 0.41 & 17 \\
\hline 7.BC possible that threatens your future educational and practical & 1.27 & 3.5 & 0.36 & 11 \\
\hline 8.BC adversely affects the physical conditions of the patient & 1.24 & 3.2 & 0.39 & 15 \\
\hline 9.BC threatens the marital and family relations & 1.23 & 3.3 & 0.37 & 12 \\
\hline 10.BC as possible causes of death in the absence of early treatment & 1.01 & 4.1 & 0.25 & 7 \\
\hline Subscale items statistics & 1.14 & 3.6 & & \\
\hline \multicolumn{5}{|l|}{ Perceived benefit } \\
\hline 11.BSE procedure is useful to those who are in same age & 939 & 4.1 & 0.23 & 6 \\
\hline 12.BSE helps early detection of $B C$ & 888 & 4.3 & 0.20 & 2 \\
\hline 13.Early detection leads less surgical intervention and best healing & 952 & 4.2 & 0.23 & 5 \\
\hline 14.BSE is an effective method for detecting cancer & 875 & 4.0 & 0.21 & 3 \\
\hline 15.Procedural BSE regularly it reduces the fear of $B C$ & 921 & 4.0 & 0.23 & 4 \\
\hline Subscale items statistics & 915 & 4.1 & & \\
\hline \multicolumn{5}{|l|}{ Perceived barriers } \\
\hline 16.I do not find time to perform BSE & 1.11 & 2.9 & 0.38 & 14 \\
\hline 17.I do not see the importance to conduct BSE & 1.06 & 2.2 & 0.47 & 21 \\
\hline
\end{tabular}




\begin{tabular}{|c|c|c|c|c|}
\hline Items & $\begin{array}{l}\text { Standard } \\
\text { Deviation }\end{array}$ & Mean & $\begin{array}{l}\text { Coefficient of } \\
\text { Variance }\left(C_{V}\right)\end{array}$ & Rank \\
\hline 18.I do not know the correct way to conduct BSE & 1.25 & 3.6 & 0.35 & 10 \\
\hline 19.BSE increases my anxiety and fear & 1.33 & 3.1 & 0.42 & 18 \\
\hline 20. Not conducted BSE because of fear of cancer is detected & 1.24 & 2.7 & 0.46 & 20 \\
\hline 21.I do not check because my age, is an exhibition of cancer & 1.18 & 2.3 & 0.49 & 24 \\
\hline 22.I do not check because no one from the family has had cancer & 1.20 & 2.4 & 0.48 & 22 \\
\hline 23.I do not check for that defect & 1.04 & 1.9 & 0.54 & 26 \\
\hline 24.I do not check because it is discomfort & 1.01 & 2.0 & 0.49 & 23 \\
\hline 25.I do not check because of laziness & 1.10 & 2.2 & 0.49 & 25 \\
\hline 26.I do not check because the leave the condition on God & 1.30 & 3.0 & 0.43 & 19 \\
\hline Subscale items statistics & 1.16 & 2.6 & & \\
\hline
\end{tabular}

Table 3 presents the 26 items which are derived from health belief model and practice of BSE. The participants rated their belief items from strongly disagree to strongly agree, the mean and standard deviation is calculated for each item, as well as the percentage for each rating character for each item; rating characters includes strongly disagree, disagree, neutral, agree, and strongly agree. Also, the items are ranked according to the Coefficient of Variation $\left(\mathrm{C}_{\mathrm{V}}\right)$ which is more useful than the standard deviation because the standard deviation of data must always be understood in the context of the mean of the data. Instead, the actual value of the $C_{V}$ is independent of the unit in which the measurement has been taken, so it is a dimensionless number. For comparison between data sets with different units or widely different means, one should use the coefficient of variation instead of the standard deviation [20]. Based on a scale from one to five the overall perceived level was moderate $(M=3$; $\mathrm{SD}=1.07$ ) on a 5-point Likert-type scale. Generally, we can consider that items that have a mean equal or more the 3 have more agreement as perceived by the participant; those items include item number $5,12,13,10,11,14,15,4,18,1$, $7,9,8,6,19,26$; those items are arranged from the most agreed items to the less agreed items by the participants. On the other hand, items that have a mean less than 3 have less agreement as perceived by the participants; those items include item number 3, 16, 20, 22, 2, 21, 17, 25, 24, 23; those items are arranged from the most agreed items to the less agreed. In other word, the majority of participants believed that $\mathrm{BC}$ is dangerous to their health. $(M=4.4, S D=0.88)$; $(61 \%)$ are strongly agreeing, $(27 \%)$ are agreeing, $(7 \%)$ are neutral, (4\%) are disagreeing and (1\%) are strongly disagreeing on the fifth statement. The fifth statement had the lowest $C_{V}$ value $(C=0.2)$ which means that the most of the participants agreed on this statement and it was ranked as the first statement that the participants agreed on it. Also, the participants believed that BSE helps early detection of BC $(M=4.3, S D=0.88) ;(56 \%)$ are strongly agreeing, $(27 \%)$ are agreeing, (13\%) are neutral, $(2 \%)$ are disagreeing and $(1 \%)$ are strongly disagreeing on the twelfth statement. The twelfth statement had $C_{V}$ value of 0.201 which means that it is the next most agreed statement by participants and it was ranked as the second statement that the subjects agreed on it. The Thirteenth item which states that BSE is an effective method for detecting cancer was also perceived positively the participants $(M=4.2, S D=.95) ;(50 \%)$ are strongly agreeing, (26\%)are agreeing, (19\%) are neutral, (3\%) are disagreeing and $(2 \%)$ are strongly disagreeing on the third statement. Statement (13) had a low $\mathrm{C}_{\mathrm{V}}$ value of 0.23 which means that large number of the participants agreed on this statement and it was ranked as the third statement that the subjects agreed on it. The least agreed item was statement (23) $(M=1.9$, $S D=1.04) ;(4 \%)$ are strongly agreeing, (3\%) are agreeing, $(16 \%)$ are neutral, (34\%) are disagreeing and $(41 \%)$ are strongly disagreeing on the statement.

Table 4. Sources of information about breast cancer $(n=917)$.

\begin{tabular}{lll}
\hline Source of information & No & $\mathbf{\%}$ \\
\hline Media & 295 & 32 \\
Books \& Magazines & 34 & 4 \\
School & 66 & 7 \\
Working in the field of health & 84 & 9 \\
Other Sources (publications, internet, Friends \& & 234 & 26 \\
Community) & 204 & 22 \\
More than one source (all of the above) & & \\
\hline
\end{tabular}

Table 4 profiles the sources of information about $\mathrm{BC}$ as perceived by secondary school students. $32 \%$ of participants considered media as a major source of information about $\mathrm{BC}$, 26\% considered other sources (publications, internet, friends and community, $9 \%$ considered working in the field of health as a source of information, $7 \%$ considered school as a source of information about $\mathrm{BC} 4 \%$ considered books and magazines as a source of information and $22 \%$ considered more than one of the mentioned above sources for information about BC.

\section{Discussion}

Health behaviors that are formed during adolescence can enhance future health and have indications for the entire life course. This study set out with the aim to determine the perception level of female adolescent about $\mathrm{BC}$ and breast self-exam. It is one of the studies in SA that aim to assess the perception of Saudi female adolescent toward BC in Riyadh city. A study conducted in SA estimated that the future burden of BC in SA is expected to increase by approximately $35 \%$ by 2025 [21]. In SA limited studies about Saudi female adolescent perception of BC. Despite increasing awareness about the Breast cancer the low perception about $\mathrm{BC}$ disease prevention among secondary school students are responsible for a negative perception of the curability of a cancer 
detected early and of the efficacy of the screening tests. The present results for relationship between demographic variables and perception level were broadly consistent with previous applications of the HBM in relation to BSE. Those studies have found significant effects for perceived benefits [22-23].

The women who perceived more benefits in relation BSE were more likely to engage in the behavior. Study participants level of perceived barriers to BSE was found to be negatively associated with their performance of BSE. Thus as perceived barriers decreased, the women's level of BSE increased. This finding was also consistent with findings of other studies [24-25]. Previous studies have found conflicting results about perceived benefits and barriers. For example, perceived benefits and barriers have been found no significant effects to BSE performance "perceived benefits" [26-27] "perceived barriers ". Another studies demonstrated a relation between perceived benefits and BSE performance [28-29]. In this study perceived seriousness, susceptibility was not significantly predicting the BSE performance. This observation confirms the results of previous studies [23-30]. One third of participants perceived media as a major source of information about $\mathrm{BC}$ then other sources such as publications, internet, friends and community. This result is in line with another studies [31-32] and in contrast to another study that revealed more than half of the sample used mass media [33].

Scholars used to create and implement interventions as a major means by which to disseminate knowledge and increase awareness about a given health problem, such as BC Interventions that yield behavior changes are ideally based on at least one theoretical framework. In this study the health belief model (HBM) as a cognitive model used to explain engagement (or lack of engagement) in health-promoting behavior. The HBM was one of the first models to adapt theories from the behavioral sciences in order to examine health related problems and one of the most widely recognized and used models in health behavior applications. Many investigators studying beliefs related to cancer screening practices have used the $\mathrm{HBM}$ as a theoretical framework to study BC screening behavior such as breast self-exam (BSE) or mammography screening. The health belief model has been applied to predict and explain a wide variety of health-related behaviors using four constructs (perceived susceptibility, seriousness, benefits, and barriers).

To better understand $\mathrm{BC}$ survival and outcome, early detection is the cornerstone for $\mathrm{BC}$ control by early screening. Screening is not only for the disease at the early stages by using latest technology screen machine but by assessing the female adolescent perception toward the disease process. In Saudi Arabia, BC is the most common cancer among woman, ranked first among females and accounting for $21 \%$ of all newly diagnosed female cancers [34]. It usually presents at late presentation of advanced cases and more frequently in young Saudi women. Cultural beliefs and practices play role in adolescent perception toward $\mathrm{BC}$ and $\mathrm{BSE}$ and may play a potential key barrier for early examination and primary prevention. Even though in some cultures, women may be believed that any discussion or speaking the word breast cancer can be brought upon themselves which may hinder early detection and treatment of breast cancer, the cultural background not necessary explained every aspect of health behaviors. However, constraints due to culture or religion may render or hinder the appropriate health behaviors. Culturally based and within Saudi unique culture it is important to understand different health behaviors that can be identified and develop health protection interventions for attitudinal change which could increase in desirable health behavior for adolescent age groups. This avenue should be explored more for its potential role in health education and primary prevention. Moreover, given the strong predictive utility of age groups should seek to give us insight about the importance of targeting female adolescent in high school due to increase awareness of students with age advancement.

\section{Conclusion and Recommendation}

This study identified the perception of female adolescent in secondary high school about $\mathrm{BC}$ and provided an important baseline data about perception of female adolescent at age group of 15-20 years. In sum, findings of the study showed that the participant perception level about BC and BSE was moderate. Primary prevention and community care is the concern now in Saudi health care sectors especially with Saudi 2030 vision. Encouragement from the government such as Ministry of Health through school health units have an effective role in raising awareness, knowledge to increase female adolescent perception about $\mathrm{BC}$ in the school environment. In particular, health professionals should continue to highlight the positive benefits of performing regular BSE as well as developing ways to address the barriers that women may experience. With the chances of unexpected $\mathrm{BC}$ cases increase due to rapid increase in the population number of Saudi women, BC control program is required. Nurses are required to assess for female adolescent perception about $\mathrm{BC}$ which in later could help to develop an effective $\mathrm{BC}$ control programs for female adolescence.

It is highly recommended for replication of the study in other regions with different sample and in different culture groups within the kingdom. It is also important to note that this study did not include clinical breast examination as one of its areas of focus because breast self-exam and clinical breast exam are the two main forms of $\mathrm{BC}$ screening behaviors recommended for women in this age group, future research should examine both behaviors in terms of $\mathrm{BC}$ screening compliance. Although this study provided valuable information about BSE frequencies and BC perception levels for female adolescent, it was limited to provide in-depth information about the cultural issue. Further studies are needed to explore the effect of age on the performance of female adolescent and using qualitative methodology to explore for cultural issue and BSE practice. 


\section{References}

[1] Fitzmaurice C, Allen C, Barber R, Barregard L, Bhutta Z, Brenner $\mathrm{H}$ et al. Global, Regional, and National Cancer Incidence, Mortality, Years of Life Lost, Years Lived With Disability, and Disability-Adjusted Life-years for 32 Cancer Groups, 1990 to 2015. JAMA Oncology. 2017;3(4):524.

[2] About Breast Cancer [Internet]. American Cancer Society (ACS). 2017 [cited 16 May 2017]. Available from: https://www.cancer.org./content/dam/CRC/PDF/Public/8577.0 $0 . p d f$

[3] Invasive Cancer Incidence-United States, 2009. JAMA. 2013;309(15):1579 .

[4] King's College London - The global burden of cancer [Internet]. Kcl.ac.uk. 2016 [cited 16 May 2017]. Available from:

http://www.kcl.ac.uk/newsevents/news/newsrecords/2016/11 $\% 20$ November-/Global-burden-of-cancer.aspx

[5] Ginsburg O, Bray F, Coleman M, Vanderpuye V, Eniu A, Kotha $\mathrm{S}$ et al. The global burden of women's cancers: a grand challenge in global health. The Lancet. 2017;389(10071):847860 .

[6] World Health Organization (WHO). . Breast cancer: prevention and control [Internet]. 2013. Available from: http://www.who.int/cancer/detection/breastcancer/en/

[7] Al-Haji K, Moawed S. Breast Cancer, Breast Self Examination Knowledge among Female High School Students in Riyadh City. Middle East Journal of Nursing. 2015;9(1):2533.

[8] POPULATION ESTIMATES [Internet]. General authority for statistics (GAS). 2017 [cited 16 May 2017]. Available from: https://www.stats.gov.sa/en/43>

[9] Bishop A, Baker G, Boyle T, MacKinnon N. Using the Health Belief Model to explain patient involvement in patient safety. Health Expectations. 2015;18(6):3019-3033.

[10] Farajzadegan Z, Rouzbahani R, Fathollahi-Dehkordi F, Tavakoli $\mathrm{N}$ et al. The transtheoretical model, health belief model, and breast cancer screening among Iranian women with a family history of breast cancer. Journal of Research in Medical Sciences. 2016;21(8):1-8.

[11] Kobrin S, Ferrer R, Meissner H, Tiro J, Hall K, ShmueliBlumberg D et al. Use of Health Behavior Theory in Funded Grant Proposals: Cancer Screening Interventions as a Case Study. Annals of Behavioral Medicine. 2015;49(6):809-818.

[12] $\mathrm{Wu} \mathrm{T,} \mathrm{Yu} \mathrm{M.} \mathrm{Reliability} \mathrm{and} \mathrm{Validity} \mathrm{of} \mathrm{the} \mathrm{Mammography}$ Screening Beliefs Questionnaire Among Chinese American Women. Cancer Nursing. 2003;26(2):131-142.

[13] Petro-Nustus W, Mikhail B. Factors Associated with Breast Self-Examination Among Jordanian Women. Public Health Nursing. 2002;19(4):263-271.

[14] Gürsoy A, Yı̆̆ıtbaş Ç, Yilmaz F, Erdöl H, Kobya Bulut H, Mumcu $\mathrm{H}$ et al. The effects of peer education on university students' knowledge of breast self-examination and health beliefs. Journal of Cancer Education. 2009;24(4):331-333.

[15] Katapodi M, Dodd M, Facione N, Humphreys J, Lee K. Why
Some Women Have an Optimistic or a Pessimistic Bias About Their Breast Cancer Risk. Cancer Nursing. 2010;33(1):64-73.

[16] Özgül K, Aklime D, Arzu Tuna M. Effects of peer and group education on knowledge, beliefs and breast self-examination practice among university students in Turkey. Turkish Journal of Medical Sciences. 2017;39(1):59-66.

[17] CHAMPION V. Instrument Refinement for Breast Cancer Screening Behaviors. Nursing Research. 1993;42(3):139-143.

[18] Han Y, Williams R, Harrison R. Breast cancer screening knowledge, attitudes, and practices among Korean American women. Oncology nursing forum. 1999;27(10):1585-1591.

[19] Jirojwong S, Manderson L. Beliefs and Behaviors About Pap and Breast Self-Examination Among Thai Immigrant Women in Brisbane, Australia. Women \& Health. 2001;33(3-4):53-73.

[20] Polit D, Beck C. Nursing research:Principles and Methods. 7th ed. Philadelphia: Lippincott Williams \& Wilkins; 2004.

[21] Yousuf S. Breast Cancer Awareness among Saudi Nursing Students. Journal of King Abdulaziz University-Medical Sciences. 2010;17(3):67-78.

[22] Faronbi J, Abolade J. Breast self-examination practices among female secondary school teachers in a rural community in Oyo State, Nigeria. Open Journal of Nursing. 2012;02(02):111-115.

[23] Noroozi A, Jomand T, Tahmasebi R. Determinants of Breast Self-Examination Performance Among Iranian Women: An Application of the Health Belief Model. Journal of Cancer Education. 2010; 26(2):365-374.

[24] Hajian S, Vakilian C, Najmabadi K, Hosseini J, Mirzaei H. 122 Effects of Education Based on the Health Belief Model (HBM) on Screening Behaviors in High Risk Women for Breast Cancer, Tehran, Iran. European Journal of Cancer. 2012; 48:S76

[25] Tavafian S, Hasani L, Aghamolaei T, Zare S, Gregory D. Prediction of breast self-examination in a sample of Iranian women: an application of the Health Belief Model. BMC Women's Health. 2009; 9(1):1472-6874.

[26] Donnelly T, Al Khater A, Al-Bader S, Al Kuwari M. Arab Women's Breast Cancer Screening Gaballah I. Awareness, Knowledge and Practice of Breast Self-Examination Among Saudi Women. Med. J. Cairo Univ.2011; 79(2): 8186 .Available at : www.medicaljournalofcairouniversity.com

[27] Practices: A Literature Review. Asian Pac $J$ Cancer Prev.2013; 14 (8): 4519-4528.

[28] Phumla P. An investigation of knowledge and practice of breast self-examination among female high school learners: an intervention study [the Degree of Master of Arts (Clinical Psychology)]. Department of Psychology University of Zululand; 2011.

[29] Akhtari-Zavare M. Knowledge on breast cancer and practice of breast self-examination among selected female university students in Malaysia. Medical and Health Science Journal. 2011; 7:49-56.

[30] Charkazi A, Samimi A, Razzaghi K, Kouchaki G, Moodi M, Meirkarimi $\mathrm{K}$ et al. Adherence to Recommended Breast Cancer Screening in Iranian Turkmen Women: The Role of Knowledge and Beliefs. ISRN Preventive Medicine. 2013; 2013:1-8. 
[31] Nelson, O., \& Salawu, A. Effective Use of Media Awareness Campaigns for Breast Cancer Care among Women: A Comparative Study. Journal of International Women's Studies.2016; 17(4): 160-173. Available at: http://vc.bridgew.edu/jiws/vol17/iss4/12

[32] Nelson O, Kehinde O, Oyero O, Wiliams O, Soola E. Effective Use of Information Sources for Breast Cancer Care: Interplay of Mass Media and Interpersonal Channels. Journal of African Research in Business \& Technology. 2014;:1-12.
[33] Ayed A, Harazneh L, Fashafsheh I, Nazzal S, Talahmeh B, Hajar D et al. Breast Self-Examination in Terms of Knowledge, Attitude, and Practice among Nursing Students of Arab American University/ Jenin. Journal of Education and Practice. 2015;6(4):37-47.

[34] Habib F, Salman S, Safwat M, Shalaby S. Awareness and Knowledge of Breast Cancer Among University Students in Al Madina Al Munawara Region. Middle East Journal of Cancer. 2010;4(1):159-166. 